\title{
CARACTERIZACIÓN DE PACIENTES CON COVID-19 GRAVE ATENDIDOS EN UN HOSPITAL DE REFERENCIA NACIONAL DEL PERÚ
}

\author{
Giancarlo Acosta (iD) 1,a, Gerson Escobar (iD) ${ }^{1, a}$, Gissela Bernaola (iD) ${ }^{1, a}$, Johan Alfaro (iD), \\ Waldo Taype (iD) ${ }^{1, \mathrm{a}}$, Carlos Marcos (iD ${ }^{1, \mathrm{~b}}$, Jose Amado (D) ${ }^{1,2, \mathrm{~b}, \mathrm{c}}$ \\ ${ }^{1}$ Hospital Nacional Edgardo Rebagliati Martins, Lima, Perú. \\ ${ }^{2}$ Universidad Nacional Mayor de San Marcos, Lima, Perú. \\ ${ }^{a}$ Especialista en Medicina de Emergencias y Desastres; ${ }^{b}$ especialista en Medicina Interna; ${ }^{c}$ doctor en Medicina
}

\section{RESUMEN}

Con el objetivo de describir las manifestaciones de pacientes con enfermedad por coronavirus 2019 (COVID-19), se evaluaron variables sociodemográficas, antecedentes, manifestaciones clínicas y radiológicas, tratamientos y evolución en pacientes que ingresaron por emergencia, del 6 al 25 de marzo de 2020, al Hospital Nacional Edgardo Rebagliati Martins en Lima. Se registraron 17 pacientes: el 76\% eran varones, edad promedio de 53,5 años (rango de 25 a 94); el 23,5\% había regresado del extranjero; 41,2\% referido de otros establecimientos de salud; $41,2 \%$ ingresó a ventilación mecánica; falleció el 29,4\% (5 pacientes). Los factores de riesgo detectados fueron adulto mayor, tener hipertensión arterial y obesidad; los principales síntomas, tos, fiebre y disnea; los hallazgos de laboratorio frecuentes, proteína $\mathrm{C}$ reactiva elevada y linfopenia; la presentación radiológica predominante, el infiltrado pulmonar intersticial bilateral. Se reporta una primera experiencia en el manejo de pacientes con diagnóstico de la COVID-19 grave en el Perú.

Palabras clave: Síndrome Respiratorio Agudo Grave; Neumonía Viral; Servicios Médicos de Urgencias; Virosis; Infecciones por Coronavirus; Pandemias (fuente: DeCS BIREME).

\section{DESCRIPTION OF PATIENTS WITH SEVERE COVID-19 TREATED IN A NATIONAL REFERRAL HOSPITAL IN PERU}

\begin{abstract}
In order to describe manifestations from patients with coronavirus disease 2019 (COVID-19), sociodemographic variables such as, previous medical history, clinical and radiological manifestations, treatments and evolution of patients were evaluated. This took place from March 6th to 25th, 2020, in the "Edgardo Rebagliati Martins" National Hospital in Lima. Seventeen patients were registered: 76\% were male, with an average age of 53.5 years (range 25-94); $23.5 \%$ had returned from abroad; $41.2 \%$ were referred from other health facilities; $41.2 \%$ were admitted to mechanical ventilation; $29.4 \%$ (5 patients) died. The risk factors detected were: advanced age, arterial hypertension and obesity. The main symptoms detected were: cough, fever and dyspnea. Frequent laboratory findings were: elevated C-reactive protein and lymphopenia. The predominant radiological presentation was bilateral interstitial lung infiltrate. A first experience in the management of patients diagnosed with severe COVID-19 in Peru is reported.
\end{abstract}

Citar como: Acosta G, Escobar G, Bernaola G, Alfaro J, Taype W, Marcos $\mathrm{C}$, et al. Caracterización de pacientes con COVID-19 grave atendidos en un hospital de referencia nacional del Perú. Rev Peru Med Exp Salud Publica. 2020;37(2):253-8. doi: https://doi. org/10.17843/rpmesp.2020.372.5437

Correspondencia: José Amado Tineo; jpamadot@gmail.com

Recibido: $29 / 03 / 2020$

Aprobado: 08/04/2020

En línea: 16/04/2020
Keyword: Severe acute respiratory syndrome; Pneumonia, viral; Emergency medical services; Virus diseases; Coronavirus infection; Pandemics (source: MeSH NLM).

\section{INTRODUCCIÓN}

La nueva enfermedad asociada al coronavirus, originada en China en 2019 y denominada enfermedad por coronavirus de 2019 (COVID-19, por sus siglas en inglés), es causada por un nuevo virus, llamado coronavirus de tipo 2 causante del síndrome respiratorio agudo grave (SARS-CoV-2, por sus siglas en inglés). Se caracteriza por su contagio directo, aparición de neumonía grave y peor evolución en adultos mayores o pacientes con comorbilidades como hipertensión, obesidad, diabetes o inmunosupresión, y por su rápida diseminación en el mundo ${ }^{(1,2)}$, siendo considerada pandemia por la Organización Mundial de la Salud el 11 de marzo del $2020^{(3,4)}$. 
El 6 de marzo de 2020, se confirmó la primera persona infectada en el Perú y se incrementó rápidamente el número de casos sospechosos y las primeras hospitalizaciones. El 19 de marzo se presentaron las primeras tres víctimas mortales ${ }^{(1,3)}$. $\mathrm{Al} 2$ de abril se tomaron 16518 pruebas diagnósticas y se obtuvieron 1414 casos positivos para SARS-CoV-2; 189 hospitalizados, 51 en cuidados intensivos y 41 fallecidos. Se reportó que en el mundo hubo más de un millón de infectados y más de 50000 fallecidos, siendo los países más afectados Italia, España y en los últimos días EE. UU. En América del Sur, Brasil y Ecuador son los países con más fallecidos ${ }^{(5,6)}$.

El Hospital Nacional Edgardo Rebagliati Martins, que brinda atención a los afiliados al Seguro Social de Salud (EsSalud) del Perú, es un centro de tercer nivel de complejidad designado como hospital de referencia para pacientes COVID-19. El presente estudio busca describir las manifestaciones clínicas en pacientes atendidos por COVID-19 grave en el servicio de emergencia de este hospital nacional.

\section{EL ESTUDIO}

Se realizó un estudio observacional retrospectivo en el servicio de emergencia de adultos del Hospital Nacional Edgardo Rebagliati Martins, ubicado en el distrito de Jesús María en Lima, Perú. Se define caso grave de COVID-19 a los pacientes que requirieron hospitalización o fallecieron por la enfermedad y resultado positivo para SARS-CoV-2 mediante análisis de reacción en cadena de polimerasa con transcriptasa inversa (RT-PCR, por sus siglas en inglés) de hisopado faríngeo o nasal, procesados en el Instituto Nacional de Salud.

Se incluyeron los casos presentados entre el 6 y el 25 de marzo de 2020. El 6 de marzo, fecha en que se informó el primer caso confirmado en el Perú, se atendieron en el hospital pacientes sospechosos que días después tuvieron resultados positivos. Se excluyeron los casos con manifestaciones clínicas, pero prueba molecular negativa para el virus.

Se destinó un área en el hospital para identificar, aislar y atender pacientes COVID-19. En la puerta del servicio de emergencia se ubicó un trabajador de salud capacitado (enfermero o técnico de enfermería) quien preguntaba el motivo de consulta del paciente y si tenía algún síntoma respiratorio agudo lo derivaba al área de triaje respiratorio.

El puesto de triaje respiratorio se ubicó en un área independiente del hospital, en donde un médico evaluaba al paciente sospechoso, según el flujograma institucional (Figura 1). Diariamente pasaban por el puesto de triaje alrededor de $150 \mathrm{pa}$ cientes. Utilizando la saturación de oxígeno capilar periférica, la escala de evaluación de insuficiencia orgánica secuencial rápida (qSOFA, por sus siglas en inglés) ${ }^{(7)}$ y los factores de riesgo para esta enfermedad mencionados en la norma técnica peruana, se decidía si el paciente era enviado a casa para su aislamiento, si pasaba al tópico de emergencia o si era admitido en una sala

\section{MENSAJES CLAVE}

Motivación para realizar el estudio: Ante la emergencia por el nuevo coronavirus en el Perú, conocer las manifestaciones clínicas de gravedad permitirá identificar el pronóstico de los pacientes hospitalizados.

Principales hallazgos: De 17 pacientes con COVID-19, 7 ingresaron a ventilación mecánica y 5 fallecieron. Los factores de riesgo fueron ser adulto mayor, hipertensión arterial y obesidad. Se usó azitromicina desde el inicio, la hidroxicloroquina se usó posteriormente, cuando se tuvo autorización para su uso.

Implicancias: Se reporta una primera experiencia en el manejo de pacientes adultos caracterizados por neumonía bilateral como manifestaciones graves de la COVID-19 en el Perú.

del servicio para pacientes sospechosos, confirmados o críticos (dependientes de unidad de cuidados intensivos o ventilación mecánica) ${ }^{(1,8)}$.

Se revisaron historias clínicas virtuales, resultados del laboratorio y del sistema de radiodiagnóstico del hospital. De estas fuentes se identificaron variables sociodemográficas, antecedentes, comorbilidades, manifestaciones clínicas y exámenes de laboratorio al ingreso hospitalario, se revisaron los estudios radiológicos realizados a cada paciente, el tratamiento administrado, la evolución y la estancia hospitalaria hasta el término del estudio. Como factores de riesgo se consideraron los establecidos en la norma técnica del Ministerio de Salud.

Los datos fueron digitados y procesados en Microsoft Excel 2013. Se realizó un análisis estadístico descriptivo, mediante medidas de tendencia central y distribución de frecuencias. Los datos se tomaron de fuentes secundarias y fueron codificados para asegurar la confidencialidad y el anonimato; solo los investigadores tuvieron acceso, previa autorización de la jefatura del servicio del hospital.

\section{LOS HALLAZGOS}

Se identificaron 17 pacientes con manifestaciones graves de COVID-19; la edad promedio fue de 53,5 años con un rango de 25 a 94; el $76 \%$, de sexo masculino; el 88,2\%, habitaba en distritos de Lima Centro y Sur (Miraflores, Jesús María, San Borja, San Isidro, Surquillo, Breña, Rímac, Villa María del Triunfo, Chorrillos y San Juan de Miraflores) y un paciente radicado en los EE. UU. Tres pacientes adquirieron la infección en Europa (llegados de España, Inglaterra y Holanda) y uno en los EE. UU. El 76\% (13 pacientes) tuvo al menos un factor de riesgo para desarrollar infección grave por COVID19, otros factores de riesgo incluyen corticoterapia crónica, gestación e infección por virus de inmunodeficiencia humana. El 


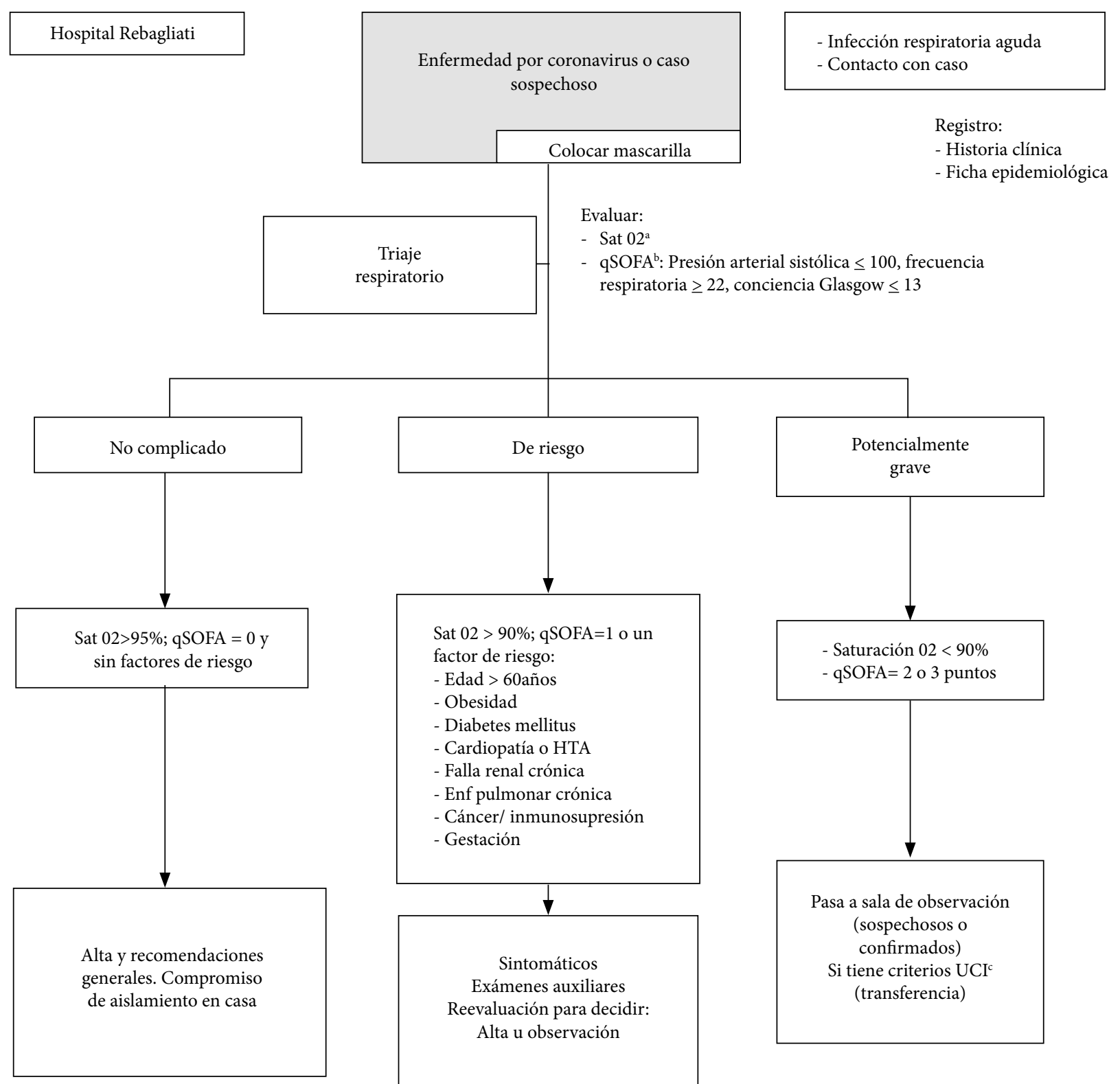

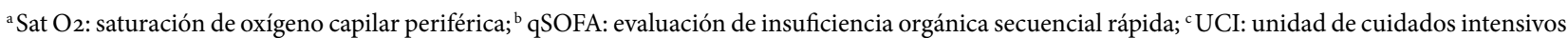
Adaptado de documentos técnicos del Ministerio de Salud de Perú y España.

Figura 1. Flujograma de atención de pacientes con COVID-19 confirmada o sospechosa en el servicio de emergencia de un hospital nacional de Lima, Perú, en marzo de 2020.

23,5\% (4 pacientes) tuvo más de un factor de riesgo a la vez (adulto mayor más hipertensión arterial o diabetes). Cuatro pacientes fueron transferidos de clínicas privadas, dos de otro establecimiento de EsSalud y uno de un hospital del Ministerio de Salud, todos ingresaron por triaje respiratorio y fueron derivados a una sala asignada (Tabla 1).

Los pacientes ingresaron al hospital con un tiempo de enfermedad de 7 días, dentro de un rango de 3 y 13 días. Los síntomas más frecuentes fueron tos, fiebre y disnea; los signos fueron estertores respiratorios y polipnea; los hallazgos de laboratorio fueron elevación de proteína C reactiva y linfope- nia. El hallazgo radiológico más frecuente fue el infiltrado pulmonar intersticial bilateral (Tabla 2), no se encontraron imágenes de derrame pleural. Solo se realizó tomografía a un paciente, cuando no tenía diagnóstico confirmatorio y por dolor abdominal, y se encontraron lesiones focales bilaterales en vidrio esmerilado en lóbulos pulmonares inferiores (Figura 2). En dos pacientes no se realizaron análisis de laboratorio y en cuatro no se tomaron radiografías por deficiencias logísticas en los primeros días.

Fueron 13 los pacientes que recibieron algún tipo de tratamiento antimicrobiano; la hidroxicloroquina estuvo 
Tabla 1. Características epidemiológicas de pacientes graves por SARS-CoV-2 en un hospital nacional de Lima, Perú, en marzo de 2020.

\begin{tabular}{lcc}
\hline Características & $\mathbf{n = 1 7}$ & $\mathbf{\%}$ \\
\hline Grupo etario (años) & 1 & 5,9 \\
Menor de 30 & 5 & 29,4 \\
de 30 a 39 & 1 & 5,9 \\
de 40 a 49 & 4 & 23,5 \\
de 50 a 59 & 6 & 35,3 \\
60 o más & & \\
Sexo & 13 & 76,5 \\
Masculino & 4 & 23,5 \\
Femenino & & \\
Distrito de procedencia en Lima & 11 & 64,7 \\
Lima Centro & 4 & 23,5 \\
Lima Sur & 2 & 11,8 \\
Otros & & \\
Historia de viajes o contactos & 4 & 23,5 \\
Viaje reciente a países con COVID 19 & 3 & 17,6 \\
Contactos confirmados & 10 & 58,9 \\
Contactos no conocidos & 7 & 41,2 \\
Referidos de otros centros & & \\
Factores de riesgo & 6 & 35,3 \\
Adulto mayor & 4 & 23,5 \\
Hipertensión arterial & 3 & 17,6 \\
Obesidad & 2 & 11,8 \\
Diabetes mellitus & 2 & 11,8 \\
Enfermedad renal crónica & 3 & 17,6 \\
Otros & & \\
\hline
\end{tabular}

COVID-19: enfermedad por coronavirus de 2019

disponible 3 días antes del cierre de la recolección de datos de la presente investigación. Todos los pacientes recibieron oxígeno suplementario y el 41,2\% (7) ingresó a ventilación mecánica.

De los 5 pacientes fallecidos, 4 eran varones (80\%), tres mayores de 70 años de edad, y 2 habían venido de España, donde adquirieron la infección. Un paciente de 38 años llegó con insuficiencia respiratoria grave y falleció diez horas después de ser intubado y colocado en un ventilador mecánico. Un paciente no fue hospitalizado y falleció dos días después de habérsele tomado el hisopado faríngeo, manifestó dificultad respiratoria grave antes del deceso en su domicilio.

Dos pacientes sin factores de riesgo fueron dados de alta después de 2 y 4 días respectivamente, por evolución favorable. Al término del estudio cinco pacientes permanecían en cuidados intensivos en ventilación mecánica, con estancia promedio de 7,3 días (entre 4 y 14); presentaron como complicaciones falla renal aguda con hemodiálisis de apoyo (dos casos), shock distributivo en pacientes que estaban recibiendo apoyo inotrópico (dos casos), falla hepática moderada (dos casos) y plaquetopenia moderada (un caso).

En sala general, permanecieron 5 pacientes con evolución estacionaria y estancia promedio de 2 días (entre 1 y 5 ). Una
Tabla 2. Manifestaciones clínicas, exámenes auxiliares y tratamiento de pacientes con enfermedad grave por virus SARSCoV-2 en un hospital nacional de Lima, Perú, en marzo de 2020

\begin{tabular}{|c|c|c|}
\hline Manifestaciones & $\mathrm{n}=17$ & $\%$ \\
\hline \multicolumn{3}{|l|}{ Síntomas y signos } \\
\hline Tos & 14 & 82,4 \\
\hline Fiebre & 13 & 76,5 \\
\hline Disnea & 13 & 76,5 \\
\hline Polipnea ( $>20$ respiraciones por minuto) & 11 & 64,7 \\
\hline Estertores respiratorios & 11 & 64,7 \\
\hline Odinofagia & 9 & 52,9 \\
\hline Sat $\mathrm{O}_{2} \mathrm{a}<90 \%$ & 5 & 29,4 \\
\hline Hipotensión & 2 & 11,8 \\
\hline Diarrea & 2 & 11,8 \\
\hline \multicolumn{3}{|l|}{ Analítica sanguínea } \\
\hline $\begin{array}{l}\text { Proteína C reactiva elevada } \\
\text { (entre } 7 \text { y } 34 \mathrm{mg} / \mathrm{dL} \text { ) }\end{array}$ & 12 & 70,6 \\
\hline Linfopenia $<900 \mathrm{~mm}^{3}$ (entre 150 y 890 ) & 11 & 64,7 \\
\hline Transaminasas elevadas (>40 U/L) & 9 & 52,9 \\
\hline Hipoxemia (paO2 < 60 mmHg) & 6 & 35,3 \\
\hline Hiperglicemia (>120 mg/dL) & 6 & 35,3 \\
\hline Leucocitosis $\left(>10000 \mathrm{~mm}^{3}\right)$ & 4 & 23,5 \\
\hline \multicolumn{3}{|l|}{$\begin{array}{l}\text { Patrones radiológicos pulmonares al ingreso } \\
(\mathrm{n}=13)\end{array}$} \\
\hline Intersticial difuso bilateral & 6 & 46,2 \\
\hline Alveolointersticial bilateral & 4 & 30,7 \\
\hline Reticulonodular bilateral & 3 & 23,1 \\
\hline \multicolumn{3}{|l|}{ Tratamiento antimicrobiano } \\
\hline Azitromicina & 7 & 41,2 \\
\hline Hidroxicloroquina & 5 & 29,4 \\
\hline Oseltamivir & 3 & 17,6 \\
\hline \multicolumn{3}{|l|}{ Otros antimicrobianos } \\
\hline Piperacilina tazobactam & 4 & 23,5 \\
\hline Meropenem y vancomicina & 2 & 11,8 \\
\hline Ceftriaxona & 2 & 11,8 \\
\hline Cefepime, cotrimoxazol o ciprofloxacino & 1 & 5,9 \\
\hline \multicolumn{3}{|l|}{ Otros medicamentos utilizados } \\
\hline Metamizol & 9 & 52,9 \\
\hline Acetilcisteina & 8 & 47,1 \\
\hline Salbutamol & 6 & 35,3 \\
\hline Acetaminofeno & 3 & 17,6 \\
\hline
\end{tabular}

Sat $\mathrm{O}_{2}$ : saturación de oxígeno capilar periférica

paciente era una gestante de 32 semanas, recibió ceftriaxona endovenosa, paracetamol y dos días después se le realizó cesárea de emergencia con buen resultado; el recién nacido pasó a sala de aislados de neonatología y la madre permaneció en unidad de cuidados intensivos con oxígeno por máscara de reservorio.

\section{DISCUSIÓN}

Los casos graves de COVID-19 descritos en el presente estudio corresponden a los primeros atendidos en un hospital en la etapa inicial de la pandemia en el Perú. Similares a los casos 

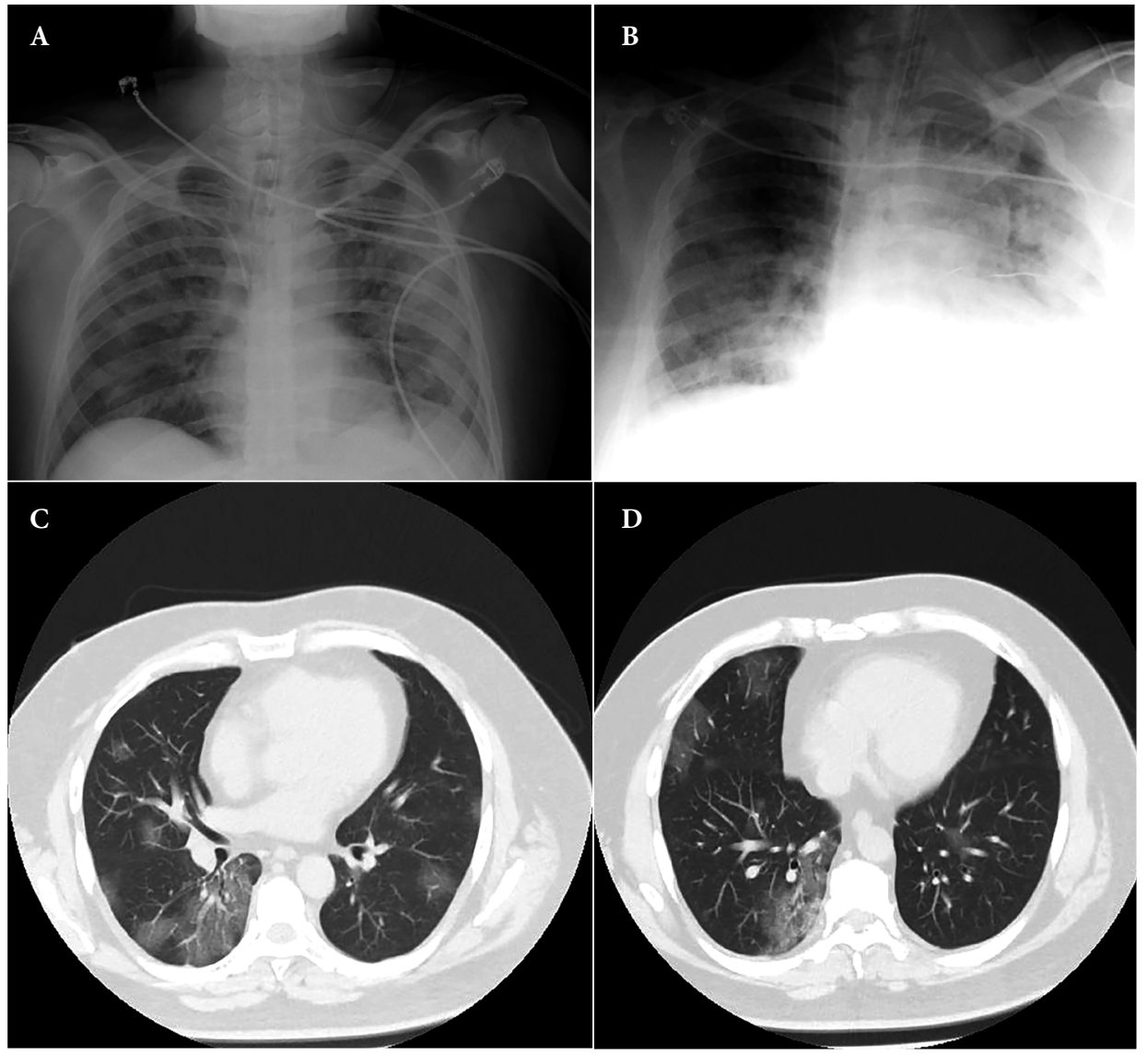

Figura 2. Pacientes con neumonía grave por SARS-CoV-2. (A) Radiografía de tórax de varón 25 años con patrón reticulonodular bilateral. (B) Radiografía de tórax de varón 47 años con patrón alveolar y broncograma aéreo bilateral. (C y D) Imágenes de tomografía de tórax de varón 53 años con lesiones focales multilobares en vidrio esmerilado bilaterales.

reportados en China y en los EE. UU., indican una forma de enfermedad predominante en varones que inicia con tos y otros síntomas respiratorios, con un tiempo de enfermedad promedio de siete días ${ }^{(10-13)}$. La fiebre y la disnea son síntomas menos frecuentes y no sería adecuado tomarlos como criterio diagnóstico o de gravedad ${ }^{(12)}$, en su lugar se proponen considerar datos objetivos, como saturación de oxígeno capilar o escores clínicos de fácil acceso para la atención inicial, y tomar acciones en el paciente ${ }^{(7)}$. Los síntomas gastrointestinales son presentaciones atípicas y poco frecuentes ${ }^{(9)}$.

La presente serie tiene solo el 35\% de casos mayores de 60 años, menor a lo reportado en China y EE. UU. para los casos graves. Esto se podría deber al menor número de adultos mayores en la población peruana, o por corresponder a la primera etapa de la epidemia, pues esta infección ingresó al país por viajes internacionales, principalmente aéreos, y por personas jóvenes. Se encuentran también comorbilidades, como hipertensión arterial y obesidad, que se mencionan como factores de riesgo para desarrollar la forma grave de enfermedad ${ }^{(9-12)}$.
Los reportes de exámenes auxiliares en COVID-19 son escasos, en nuestro estudio predomina la elevación de reactantes de fase aguda como proteína $\mathrm{C}$ reactiva y linfopenia, similar a lo informado en China o en los EE. UU. Respecto al punto de corte para considerar linfopenia, esta fue menor de 900 linfocitos por milímimetro cúbico en comparación a los 1500 por $\mathrm{mm}^{3}$ que se consideró en los EE. UU. Llama la atención la mayor frecuencia de elevación de enzimas hepáticas como las transaminasas frente a lo observado en otros países ${ }^{(9-12)}$.

En esta serie de casos, las imágenes radiológicas corresponden principalmente a radiografías torácicas simples, pero se evidencian lesiones pulmonares multifocales y bilaterales, similares a lo reportado en China y Corea ${ }^{(14-18)}$. Sin embargo, el mejor estudio para este problema es la tomografía computarizada, pues permite determinar el pronóstico y las medidas terapéuticas a aplicar, siendo recomendable ampliar su uso en este grupo de pacientes.

No existe un tratamiento probado para la COVID-19, en el presente estudio se asocian antibióticos de amplio espectro, similares a los utilizados en otros países ${ }^{(10)}$. Desde un 
inicio se utilizó azitromicina, principalmente por ser parte del tratamiento para neumonía atípica, en cambio la hidroxicloroquina se pudo emplear semanas después, cuando se tuvo autorización hospitalaria para su uso. El apoyo ventilatorio invasivo fue relativamente menor, pero es una serie pequeña de casos y al inicio se demoró su inicio.

La mortalidad en la serie presentada fue menor al 50\% encontrada en pacientes de cuidados intensivos en EE. UU. ${ }^{(12)}$, esto debido a que el presente estudio incluye pacientes de salas de emergencia y el periodo de observación fue menor. Asimismo, los problemas de organización durante los primeros días de atención en el hospital evaluado pudieron haber influido en la evolución de los pacientes, debido a la falta de exámenes hematológicos, bioquímicos y radiológicos por inconvenientes con la bioseguridad personal y ambiental. Se tuvieron varias limitaciones, entre ellas el pequeño número de casos, datos clínicos y resultados de exámenes auxiliares incompletos y criterios diagnósticos no uniformes. No se investigó infección

\section{REFERENCIAS BIBLIOGRÁFICAS}

1. Ministerio de Salud. Atención y manejo clínico de casos de COVID19. Escenario de transmisión focalizada. Lima-Perú; 2020.

2. Wu F, Zhao S, Yu B, Chen Y-M, Wang W, Song Z-G, et al. A new coronavirus associated with human respiratory disease in China. Nature. 2020;579:265-9. doi: 10.1038/s41586-020-2008-3.

3. World Health Organization. Alocución de apertura del Director General de la OMS en la rueda de prensa sobre la COVID-19 celebrada el 11 de marzo de 2020 [Internet]. Discursos del Director General de la OMS 2020 [citado el 18 de marzo de 2020 ]. Disponible en: https://www.who. int/es/dg/speeches/detail/who-director-general-s-opening-remarks-atthe-media-briefing-on-covid-19---11-march-2020.

4. Chen N, Zhou M, Dong X, Qu J, Gong F, Han Y, et al. Epidemiological and clinical characteristics of 99 cases of 2019 novel coronavirus pneumonia in Wuhan, China: a descriptive study. Lancet. 2020;395(10223):507-13. doi: 10.1016/S0140-6736(20)30211-7.

5. World Health Organization. Cumulative confirmed COVID-19 cases reported by countries and territories in the Americas [Internet]. 2020 [citado el 2 de marzo de 2020]. Disponible en: https://www.paho.org/en/ documents/cumulative-suspected-and-confirmed-covid-19-cases-reported-countries-and-territories-13.

6. Ministerio de Salud - Perú. MINSA: Casos confirmados por Coronavirus COVID-19 ascienden a 1414 en Perú Comunicado N49. 2020 [citado el 2 de abril de 2020]. Disponible en: https://www.gob.pe/institucion/ minsa/noticias/111774-minsa-casos-confirmados-por-coronavirus-covid-19-ascienden-a-1414-en-el-peru-comunicado-n-49.

7. Ramos JGR, Da Hora R, Teixeira MB, Gobatto ALN, Coutinho RVDS, Caldas JR, et al. Prognostic ability of quick-SOFA across different age groups of patients with suspected infection outside the intensive care unit: A cohort study. J Crit Care. 2018;47:178-84. doi: 10.1016/j.jcrc.2018.07.008.

8. Ministerio de Sanidad. Documento técnico Manejo en urgencias del COVID-19. Madrid; 2020. [citado el 2 de abril de 2020]. Disponible en: https://www.mscbs.gob.es/profesionales/saludPublica/ccayes/alertasActual/nCov-China/documentos/Manejo_urgencias_pacientes_con_COVID-19.pdf. concomitante con otros virus ni complicaciones bacterianas. Sin embargo, es un estudio exploratorio que aporta evidencia de los primeros casos atendidos en un hospital peruano durante la pandemia de COVID-19.

En conclusión, este estudio presenta una de las primeras experiencias, en un hospital peruano, en el diagnóstico y el tratamiento de pacientes adultos con COVID-19 grave, caracterizados por neumonía bilateral, más frecuente en varones, con comorbilidades, necesidad de oxígeno suplementario y mortalidad importante.

Contribuciones de autoría: GA, GE, GB, JA, WT, CM y JA han participado en la concepción y el diseño del artículo, aporte de pacientes, análisis e interpretación de datos, revisión crítica del artículo, aprobación de la versión final.

Conflictos de interés: Los autores declaran no tener conflictos de interés en la publicación de este artículo.

Fuente de financiamiento: Autofinanciado.

9. Zhang J, Dong X, Cao Y, Yuan Y, Yang Y, Yan Y, et al. Clinical characteristics of 140 patients infected with SARS-CoV-2 in Wuhan, China. Allergy Eur J Allergy Clin Immunol. 2020;(February):1-12. doi: 10.1111/all.14238.

10. Guan W, Ni Z, Hu Y, Liang WH, Ou CQ, He JX, et al. Clinical Characteristics of Coronavirus Disease 2019 in China. N Engl J Med. 2020 Feb 28;1-13 [Epub ahead of print]. doi: 10.1056/NEJMoa2002032.

11. Bernard S, Rolland P, Silue Y, Mailles A, Campese C, Simondon A, et al. First cases of coronavirus disease 2019 (COVID-19) in France: surveillance, investigations and control measures, January 2020. Euro Surveill. 2020;25(6). doi: 10.2807/1560-7917.ES.2020.25.6.2000094.

12. Bhatraju PK, Ghassemieh BJ, Nichols M, Kim R, Jerome KR, Nalla AK, et al. Covid-19 in Critically Ill Patients in the Seattle Region - Case Series. NEngl J Med. 2020 Mar 30; [Epub ahead of print]. doi: 10.1056/NEJMoa2004500.

13. Yang X, Yu Y, Xu J, Shu H, Xia J, Liu H, et al. Clinical course and outcomes of critically ill patients with SARS-CoV-2 pneumonia in Wuhan, China: a single-centered, retrospective, observational study. Lancet Respir Med. 2020;(20):1-7 [Epub ahead of print]. doi: 10.1016/S2213-2600(20)30079-5.

14. Shi F, Yu Q, Huang W, Tan C. 2019 Novel Coronavirus (COVID-19) Pneumonia with Hemoptysis as the Initial Symptom: CT and Clinical Features. Korean J Radiol. 2020;21:e42. doi: 10.3348/kjr.2020.0181.

15. Xu Z, Shi L, Wang Y, Zhang J, Huang L, Zhang C, et al. Pathological findings of COVID-19 associated with acute respiratory distress syndrome. Lancet Respir Med. 2020;8(4):420-422. doi: 10.1016/S2213-2600(20)30076-X.16. Shi H, Han X, Zheng C. Evolution of CT Manifestations in a Patient Recovered from 2019 Novel Coronavirus (2019-nCoV) Pneumonia in Wuhan, China. Radiology. 2020;295(1):20. doi: 10.3348/kjr.2020.0181.

17. Shi H, Han X, Jiang N, Cao Y, Alwalid O, Gu J, et al. Radiological findings from 81 patients with COVID-19 pneumonia in Wuhan, China: a descriptive study. Lancet Infect Dis. 2020;20(4):425-34. doi: 10.1016/ S1473-3099(20)30086-4.

18. Yoon SH, Lee KH, Kim JY, Lee YK, Ko H, Kim KH, et al. Chest Radiographic and CT Findings of the 2019 Novel Coronavirus Disease (COVID-19): Analysis of Nine Patients Treated in Korea. Korean J Radiol. 2020;21(4):494-500. doi: 10.3348/kjr.2020.0132. 